\title{
Transcription factor Krüppel-like factor 2 plays a vital role in endothelial colony forming cells differentiation
}

\author{
Yimeng Song ${ }^{1 \dagger}$, Xiaoxia Li ${ }^{1 \dagger}$, Dawei Wang ${ }^{1}$, Chenglai Fu' ${ }^{1}$, Zhenjiu Zhu' ${ }^{1}$, Ming-Hui Zou ${ }^{2 *}$, \\ and $\mathrm{Yi} \mathrm{Zhu}^{1,3 *}$
}

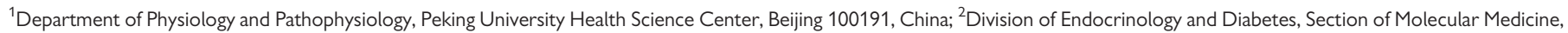
Department of Medicine, University of Oklahoma Health Sciences Center, 941 Stanton L. Young Blvd. BSEB 314, Oklahoma City, OK 73104, USA; and ${ }^{3}$ Department of Physiology and Pathophysiology, Tianjin Medical University, 22 Qixiangtai Road, Heping District, Tianjin 300070, China

Received 31 October 2012; revised 17 April 2013; accepted 5 May 2013; online publish-ahead-of-print 10 May 2013

Time for primary review: 35 days

Aims Endothelial colony forming cells (ECFCs) participate in post-natal vasculogenesis. We previously reported that vascular
endothelial growth factor (VEGF) promotes human ECFC differentiation through AMP-activated protein kinase (AMPK)
activation. However, the mechanisms underlying transcriptional regulation of ECFC differentiation still remain largely
elusive. Here, we investigated the role of transcription factor Krüppel-like factor 2 (KLF2) in the regulation of ECFC
function.

Methods $\quad H u m a n$ ECFCs were isolated from cord blood and cultured. Treatment with VEGF significantly increased endothelial and results markers in ECFCs and their capacity for migration and tube formation. The mRNA and protein levels of KLF2 were also significantly up-regulated. This up-regulation was abrogated by AMPK inhibition or by knockdown of KLF2 with siRNA. Furthermore, adenovirus-mediated overexpression of KLF2 promoted ECFC differentiation by enhancing expression of endothelial cell markers, reducing expression of progenitor cell markers, and increasing the capacity for tube formation in vitro, indicating the important role of KLF2 in ECFC-mediated angiogenesis. Histone deacetylase 5 (HDAC5) was phosphorylated by AMPK activity induced by VEGF and the AMPK agonist AICAR (5-amino- 1 - $\beta$-D-ribofuranosyl-imidazole-4-carboxamide). In vivo angiogenesis assay revealed that overexpression of KLF2 in bone-marrowderived pro-angiogenic progenitor cells promoted vessel formation when the cells were implanted in C57BL/6 mice.

Conclusion $\quad$ Up-regulation of KLF2 by AMPK activation constitutes a novel mechanism of ECFC differentiation, and may have therapeutic value in the treatment of ischaemic heart disease.

Keywords Endothelial progenitor cells • VEGF • Differentiation • AMPK • Krüppel-like factor 2

\section{Introduction}

Endothelial progenitor cells (EPCs), the precursors of endothelial cells (ECs), play an important role in the self-healing of vascular injury. These cells participate in re-endothelialization and angiogenesis and represent a promising new tool for the treatment of cardiovascular diseases. ${ }^{1}$ Since there is no clear definition for the term EPC, there are many different cell types that could fall under this definition. Key properties of these cells are colony formation in culture, adhesion at the site of injury, differentiation into ECs, and participation in angiogenesis. Hirschi et al. ${ }^{2}$ has defined this type of EPC as endothelial colony forming cells (ECFC). Many growth factors, cytokines, and chemokines have been reported to be involved in the regulation of EPC/ECFC differentiation, proliferation, and migration., 3 e had previously reported that activation of AMP-activated protein kinase (AMPK), and its consequent activation of endothelial nitric oxide synthase (eNOS) by vascular endothelial growth factor (VEGF) ${ }^{5}$ and prostaglandin E2, ${ }^{6}$ plays a pivotal role in the differentiation of this population of cells. However, the mechanisms governing regulation of transcription during EPC differentiation into mature ECs and participation in vascular repair remain largely unknown.

\footnotetext{
† These authors contributed equally to this work.

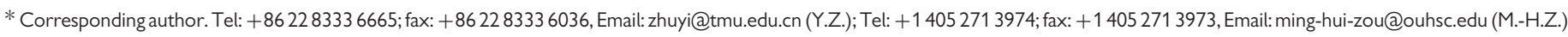
Published on behalf of the European Society of Cardiology. All rights reserved. C The Author 2013. For permissions please email: journals.permissions@oup.com.
} 
To better understand ECFC biology, we sought to identify core transcriptional factors governing ECFC differentiation. Among several transcriptional factors involved in the maintenance of EC function and cardiovascular stem/progenitor cell biology, the role of the Krüppel-like transcription factor (KLF) family in EPC biology emerged. KLFs were reported to regulate numerous biological processes, including proliferation, differentiation, development, and apoptosis. ${ }^{7}$ KLF2, also known as lung-enriched KLF, is a central regulator of endothelial function, plays a pivotal role in cardiovascular stem/progenitor cell biology, and is closely associated with endothelial homeostasis and atherogenesis. ${ }^{8,9}$ Angiopoietin-1 regulates both vascular quiescence and angiogenesis through the receptor tyrosine kinase Tie2. Angiopoietin-1/Tie2 signalling induces anti-inflammatory responses by up-regulating KLF2 through an increase in transcriptional activity of myocyte enhancer factor-2 (MEF2). ${ }^{10}$ The vascular protective effects of AMPK/SIRT1 activation induced by resveratrol and flow shear stress were reported to result from the up-regulation of KLF2 in human ECs. ${ }^{8,11}$ eNOS and thrombomodulin (TM) are known KLF2 target genes in ECs, ${ }^{12,13}$ suggesting beneficial effects of KLF2 on endothelial phenotype. Hemizygous deficiency of KLF2 increased diet-induced atherosclerosis in apolipoprotein E-deficient mice. ${ }^{14}$ Targeting modulation of the expression and/or activity of KLF2 may lead to new therapeutic approaches for cardiovascular diseases. ${ }^{15}$ Interestingly, KLF2 was recently found to have a role in cell development: KLF-binding sites were identified in the Flk1 enhancer and overexpression of KLF2 induced ectopic Flk1 expression in Xenopus embryos. ${ }^{16}$

This study aimed to investigate the potential roles and underlying mechanisms of KLF2 in EPC differentiation. Our results indicate that KLF2 plays a vital role in inducing differentiation of ECFCs into mature ECs and thereby provides new insights into the expanded regulatory circuitry in EPC-mediated angiogenesis.

\section{Methods}

\subsection{Reagents}

VEGF, basic fibroblast growth factor (bFGF), epidermal growth factor (EGF), AMPK agonist AICAR (5-amino-1- $\beta$-D-ribofuranosyl-imidazole-4-carboxamide) and antagonist Compound $C$ were from Sigma-Aldrich (St Louis, MO, USA). Insulin-like growth factor II (IGF-II) was from PeproTech (London, UK). M199 medium was from Gibco (Grand Island, NY, USA). Endothelial basal medium-2 (EBM-2) was from Lonza Clonetics (Walkersville, MD, USA), and foetal bovine serum (FBS) was from Hyclone (Logan, UT, USA). Leukaemia inhibitory factor (LIF) was from Merck Millipore (Billerica, MA, USA). Boyden chamber (6.5 mm diameter and $8.0 \mu \mathrm{m}$ pore size) was from Becton Dickinson (Franklin Lakes, NJ, USA).

\subsection{Human ECFC isolation, culture, identification, and treatment}

Human ECFC isolation, culture, and identification were previously described. ${ }^{5}$ The investigation conformed to the principles outlined in the Declaration of Helsinki for use of human umbilical cord blood. The protocol was approved by Peking University Institutional Human Sample Use Committee. Briefly, human cord blood from umbilical cords of newborns was collected with the use of heparin $(20 \mathrm{U} / \mathrm{mL})$ from donors with their written permission. Human cord blood ECFCs were isolated by density-gradient centrifugation with Ficoll $(1.077 \mathrm{~g} / \mathrm{mL})$ and plated on dishes coated with collagen type I (50 $\mu \mathrm{g} / \mathrm{mL}$; Millipore). M199 culture medium was supplemented with $20 \% \mathrm{FBS}$, human VEGF (10 $\mathrm{ng} / \mathrm{mL})$, human bFGF (1 ng/mL), human EGF $(10 \mathrm{ng} / \mathrm{mL})$, IGF II $(2 \mathrm{ng} / \mathrm{mL})$, and LIF $(10 \mathrm{ng} / \mathrm{mL})$. ECFCs at passages $2-6$ were used. For VEGF and AICAR treatments, cells were deprived of the primary culture medium containing $10 \mathrm{ng} / \mathrm{mL}$ VEGF and LIF for $12 \mathrm{~h}$, then cultured in M199 supplemented with $20 \%$ FBS and VEGF $(25 \mathrm{ng} / \mathrm{ml})$ or $\operatorname{AICAR}(1 \mathrm{mM})$ for additional 12 or $24 \mathrm{~h}$.

\subsection{Isolation and identification of mouse bone-marrow-derived pro-angiogenic progenitor cells}

Mouse bone-marrow-derived pro-angiogenic progenitor cell (BMPC) isolation, culture, and identification were as previously described. ${ }^{6}$ Briefly, BMPCs were collected by flushing the femurs and tibias of wild-type C57BL/6 mice, 8-10 weeks old, with EBM-2 medium containing 10\% FBS. Cells were plated on type I collagen-coated dishes and maintained in a humidified atmosphere containing $5 \% \mathrm{CO}_{2}$ at $37^{\circ} \mathrm{C}$. After $4-7$ days in culture, non-adherent cells were removed and adherent cells were cultured for an additional 10 days and used for further analysis. This population grew at a high proliferation rate into a monolayer of spindle-shaped cells. BMPCs at passages 2-4 were used in the following experiments. ${ }^{6} \mathrm{BMPC}$ were identified as exhibiting high levels of endothelial lineage markers CD34, c-kit, Flk-1, CD31, and VE-cadherin. Moreover, we identified their tube-forming function by their pro-angiogenic capacity to form tube-like structures on Matrigel (see Supplementary material online, Figure S1).

\subsection{Western blot analysis}

Western blot analysis was carried out according to standard protocols. Primary antibodies used were anti-p-AMPK-Thr172, anti-p-eNOS-Ser1177, anti-AMPK, and anti-eNOS (1:1000; Cell Signaling, Beverly, MA, USA); anti-p-histone deacetylase 5 (HDAC5)-Ser498 and anti-HDAC5 (1:1000; Bioworld Technology, Minneapolis, MN, USA); anti-intercellular adhesion molecule 1 (ICAM-1) and anti- $\beta$-actin (1:1000; Santa Cruz Biotechnology, Santa Cruz, CA, USA); and anti-KLF2 (1:1000; Abcam, Cambridge, MA, USA).

\subsection{Quantitative real-time polymerase chain reaction}

Total RNA was isolated from ECFCs with the use of TRlzol reagent (Invitrogen, Carlsbad, CA, USA). The resulting cDNAs were used as templates for quantitative real-time polymerase chain reaction (Q-PCR) with EVA Green fluorescent DNA stain (Biotium, Hayward, CA, USA). The primer sequences are in Table 1.

\subsection{Adenovirus infection of ECFCs and BMPCs}

Ad-KLF2, a recombinant adenovirus encoding human KLF2, was generated by subcloning cDNA encoding the total length of human KLF2 into the adenoviral vector PCMV5-Nter-FLAG. Cells cultured at $\sim 80 \%$ confluence were infected with recombinant adenovirus (50 multiplicity of infection) for $24 \mathrm{~h}$ before further treatment. The parental adenoviral vector was used as infection control.

\section{7 siRNA knockdown experiments}

KLF2 and HDAC5 siRNA and their control siRNA were synthesized by Invitrogen. Cells were plated in six-well plates and cultured for $24 \mathrm{~h}$ before transfection, then transfected with siRNA by the use of Lipofectamine RNAiMIX reagent (Invitrogen) in serum-free M199 for $4 \mathrm{~h}$, followed by addition of $10 \% \mathrm{FBS}$ and culture for an additional $48 \mathrm{~h}$ before further treatment. The forward and reverse primer sequences were human KLF2 RNAi: 5'-UCG CAC AGA UGG CAC UGG AAU GGC C-3' and 5'-GGC CAU UCC AGU GCC AUC UGU GCG A-3'; human HDAC5 RNAi: 5'-UUG UCA GGU GGU CAU GCU GUU UCU G-3' and 5' - CAG AAA CAG CAU GAC CAC CUG ACA A-3'; mouse KLF2 RNAi: 5'-UUU AGG UGC GAG CUC UUG GUG UAG G-3' and 5'-CCU ACA CCA AGA GCU CGC ACC UAA A-3'. 
Table I Primer list

\begin{tabular}{|c|c|c|c|c|c|}
\hline Human protein & $\begin{array}{l}\text { GenBank accession } \\
\text { number }\end{array}$ & Position & Product (bp) & Forward (5' to $\left.3^{\prime}\right)$ & Reverse ( $5^{\prime}$ to $\left.3^{\prime}\right)$ \\
\hline CD34 & NM_001773 & +1099 to +1459 & 360 & GCAAGCCACCAGAGCTATTC & TCCACCGTTTTCCGTGTAAT \\
\hline CD31 & NM_000442.4 & +1457 to +1629 & 173 & ATGATGCCCAGTTTGAGGTC & GACGTCTTCAGTGGGGTTGT \\
\hline eNOS & NM_000603.4 & +1402 to +1599 & 198 & ACCCTCACCGCTACAACATC & GCTCATTCTCCAGGTGCTTC \\
\hline TM & NM_000361.2 & +1028 to +1159 & 132 & GACCTCTGCGAGCACTTCTG & GGGCTCCAGTATGCAGTCAT \\
\hline$\beta$-actin & NM_007393 & +809 to +948 & 140 & GACGGCCAGGTCATCACTAT & CGGATGTCAACGTCACACTT \\
\hline
\end{tabular}

\subsection{Flow cytometry}

Fluorescence-activated cell sorting (FACS) analysis was used to detect cellsurface markers. Cells were stained for $60 \mathrm{~min}$ at $4{ }^{\circ} \mathrm{C}$, then fixed with $2 \%$ paraformaldehyde. The surface markers investigated were FITC-conjugated mouse anti-human CD34, FITC-conjugated mouse anti-human CD31, $\mathrm{PE}$-conjugated mouse anti-human c-kit, PE-conjugated mouse anti-human VE-cadherin (all from BD PharMingen). Isotype-identical antibodies served as negative controls. Analysis involved the use of FACS Calibur (Becton Dickinson) and Cell Quest software.

\subsection{Cell migration and transwell assay}

ECFC migration was assessed by scratch-wound assay as described previously. ${ }^{17} \mathrm{~A}$ wound was created in the cell monolayer, and images of cells were captured then and $8 \mathrm{~h}$ later. Images were quantified to determine the number of migrating cells.

The transwell assay was performed with a Boyden chamber. In brief, $1 \times$ $10^{5}$ cells were plated in $500 \mu \mathrm{L}$ medium in each well. M199 medium supplemented with $20 \%$ FBS was added to the upper chamber of a transwell plate and M199 medium supplemented with 20\% FBS and $100 \mathrm{ng} / \mathrm{mL}$ VEGF was added to the lower chamber. After incubation for $24 \mathrm{~h}$, cells that had migrated to the lower surface of the membrane were fixed with $2 \%$ paraformaldehyde and stained with Hoechst 33258 for 5 min. The stained cells were photographed and counted in four high-power fields per insert.

\subsection{In vitro tube-formation assay}

Matrigel (300 $\mu$ L; Becton Dickinson, Bedford, MA, USA) was added to each well of 24-well plates. Human ECFCs in M199 medium supplemented with $20 \% \mathrm{FBS}$ were then plated at $1 \times 10^{5}$ cells/well and cultured for $4 \mathrm{~h}$. After treatment with various stimuli, tube formation was examined using an inverted microscope equipped with a digital camera. Tube-like structures exceeding six cells in length were counted in five randomly selected fields in each well by three investigators blinded to the treatment.

\subsection{In vivo vasculogenesis assay}

In vivo assay of mouse BMPC angiogenesis was performed as previously described. ${ }^{6}$ The investigation conformed to the Guide for the Care and Use of Laboratory Animals by the US National Institutes of Health (NIH Publication, 8th Edition, 2011). The animal experimental protocol was approved by the Institutional Animal Care and Use Committee of the Peking University Health Science Center (LA2011-003). C57BL/6 mice were treated with ketamine $(100 \mathrm{mg} / \mathrm{kg} \mathrm{IP}) /$ xylazine $(20 \mathrm{mg} / \mathrm{kg} \mathrm{IP})$ cocktail for anaesthesia, and then BMPCs were isolated and identified (see Supplementary material online, Figure S1). BMPCs were infected with Ad-KLF2 or control virus for $24 \mathrm{~h}$ and then suspended at $5 \times 10^{5}$ cells in $100 \mu \mathrm{L}$ Matrigel on ice. The mixture was implanted on the flanks of C57BL/6 mice $(n=6)$ by subcutaneous injection. Seven days later, the euthanasia was performed with carbon dioxide and implants were removed, fixed in formalin, embedded in paraffin, and sectioned. ${ }^{6}$ Sections were stained with haematoxylin and eosin or immunohistochemically with anti-VE-cadherin to determine the presence of luminal structures.

\subsection{Statistical analysis}

The significance of variability was determined by ANOVA with post-hoc comparison, with Student's $t$-test for continuous variables and $?^{2}$ or Fisher's exact test for nominal variables, as appropriate. All data are presented as mean \pm SD from at least three independent experiments. $P<0.05$ was considered statistically significant.

\section{Results}

\subsection{Increased KLF2 expression participated in VEGF-induced ECFC differentiation}

We previously reported that VEGF-induced AMPK activation was involved in the regulation of EPC differentiation. ${ }^{5}$ Here, we treated human ECFCs with VEGF to drive ECFCs towards differentiation into mature ECs. VEGF treatment increased the phosphorylation of AMPK and eNOS in $1 \mathrm{~h}$ and the expression of the endothelial markers eNOS, ICAM-1, and CD31 within 24 h; however, the level of progenitor markers CD34 and c-kit was decreased (Figure 1A and B). We also analysed the expression of ECFC surface markers by flow cytometry. VEGF up-regulated the expressions of endothelial markers such as VE-cadherin and CD31 but down-regulated those of progenitor markers such as CD34 and c-kit (Figure 1C). ECFC capacity for cell migration and tube formation was confirmed by in vitro assays (Figure 1D-F). Furthermore, VEGF increased both the mRNA and protein levels of KLF2, which indicates that KLF2 might be involved in VEGF signalling to regulate ECFC function.

\subsection{AMPK activation was involved in ECFC differentiation via KLF2 up-regulation}

To investigate whether AMPK regulates KLF2 expression in ECFCs, we next used an AMPK agonist to activate AMPK, and its specific inhibitor to block AMPK activation. The AMPK agonist AICAR increased the levels of KLF2 mRNA and protein (Figure 2A and B) and AMPK inhibitor Compound $C$ blocked the effect of VEGF. VEGF-induced expression of the endothelial markers eNOS, ICAM-1, and CD31 was also diminished by Compound C. The progenitor markers CD34 and c-kit showed an opposite pattern (Figure 2C and D). Thus, these results suggest that KLF2 is 

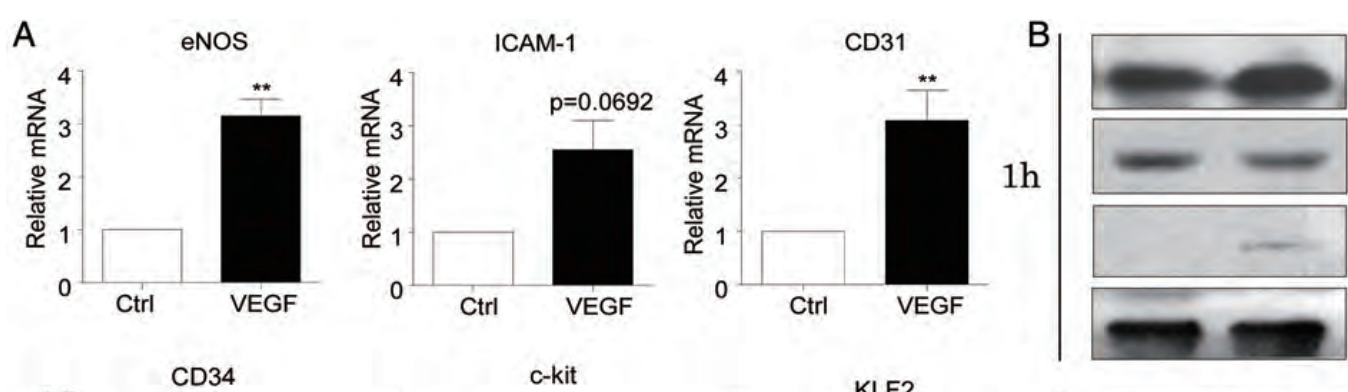

p-AMPK
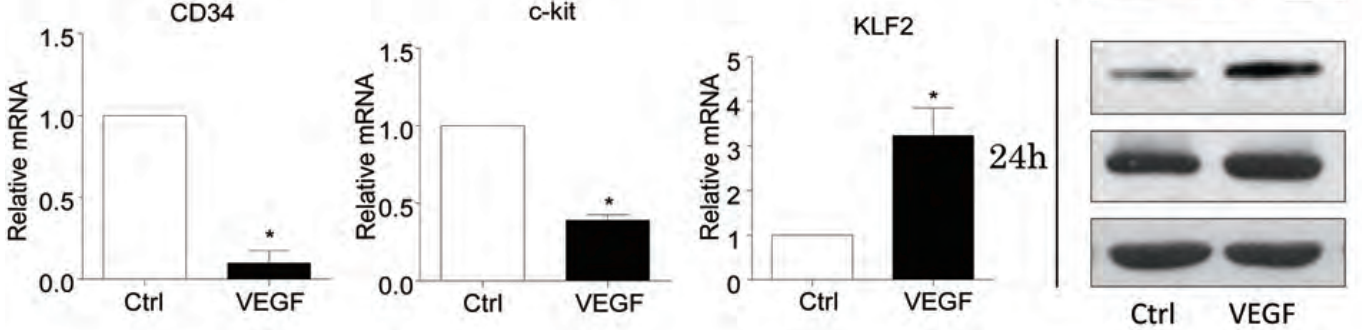

KLF2

t-AMPK

p-eNOS

t-eNOS
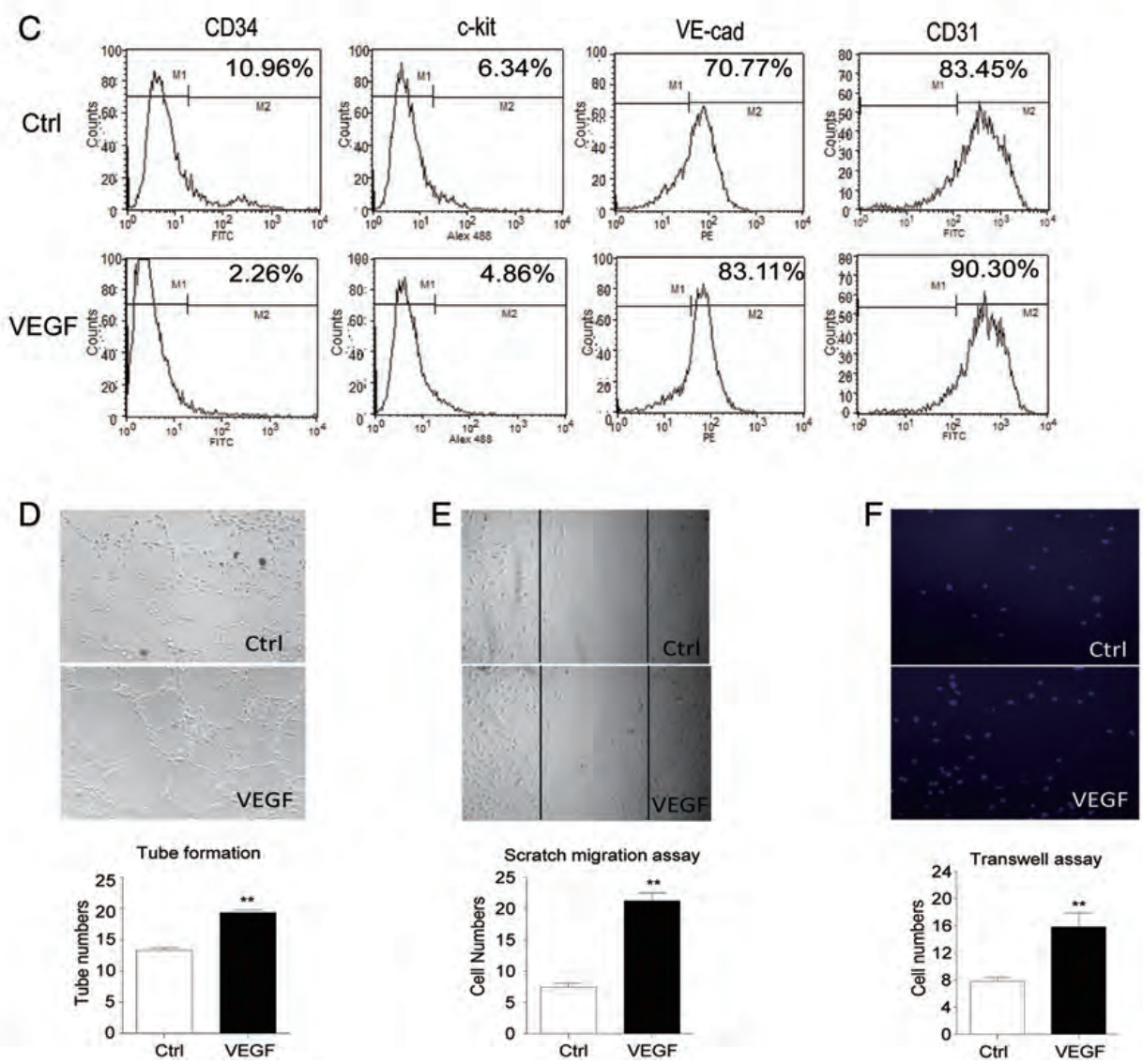

Figure I KLF2 expression was increased in VEGF-induced ECFC differentiation. Human ECFCs were treated with VEGF (25 ng/mL) for various times. (A) The mRNA levels of KLF2 and the indicated endothelial and progenitor markers were examined by Q-PCR after $12 \mathrm{~h}$ of treatment. (B) Western blot analysis of phosphorylation of AMPK and eNOS and the expression of AMPK, eNOS, and KLF2 after 1 and $24 \mathrm{~h}$, respectively. $\beta$-actin was an internal control. (C) FACS analysis of ECFCs stained with antibodies against FITC-conjugated anti-human c-kit, FITC-conjugated anti-human CD34, FITC-conjugated antihuman CD31, PE-conjugated anti-human VE-cadherin. Data are representative of three separate experiments. (D) Tube-formation assay of ECFC capillary morphogenesis after VEGF treatment. ECFCs were treated with VEGF for $24 \mathrm{~h}$ and plated on Matrigel and cultured for $4 \mathrm{~h}$. The extent of tube formation was determined by counting the tubes in five randomly chosen, low-power fields. (E) ECFCs were scratch-wounded and migrating cells were counted after 8 h. Magnification, $\times 100$. (F) Transwell assay of cells that had migrated across the filters. Magnification, $\times 100$. Data are mean \pm SD from three independent experiments, each performed in triplicate $(* P<0.05 ; * * P<0.01)$. 

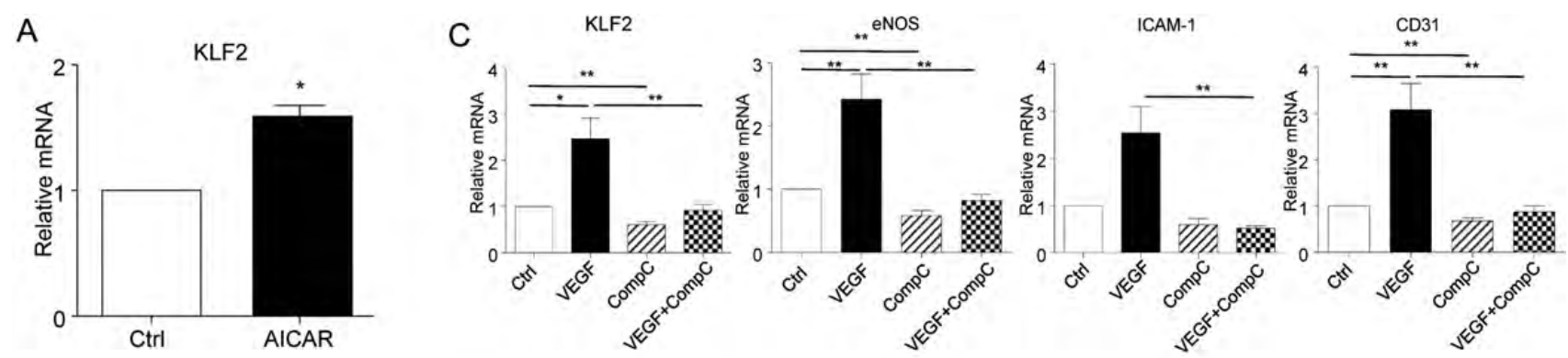

B

$1 \mathrm{~h}$

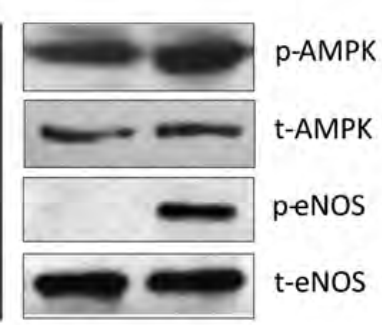

$24 \mathrm{~h}$

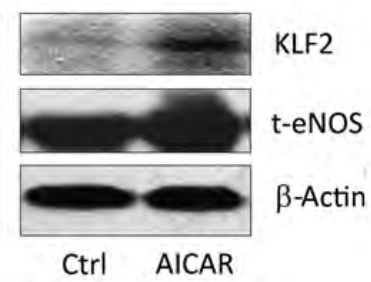

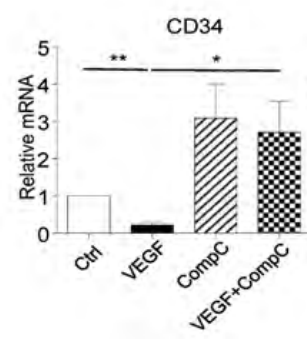

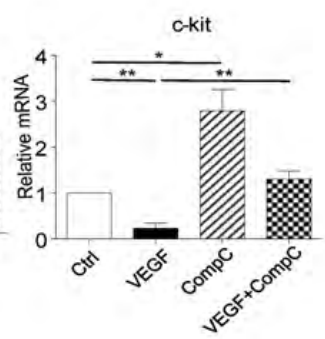

D

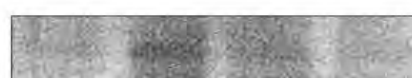

KLF2

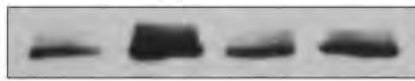

t-eNOS

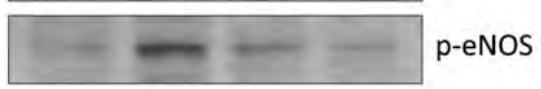

$\beta$-Actin

$\begin{array}{llllll}\text { VEGF } & - & + & & - & + \\ \text { CompC } & - & - & + & + & 24 \mathrm{~h}\end{array}$

Figure 2 AMPK was activated in ECFC differentiation via KLF2 up-regulation. ( $A$ and $B$ ) Human ECFCs were treated with AICAR for various times. KLF2 mRNA level was examined by Q-PCR after $12 \mathrm{~h}$ of treatment $(A)$. Western blot analysis of protein levels of AMPK, eNOS, and KLF2 after 1 or $24 \mathrm{~h}$ of treatment $(B)$. ( $C$ and $D)$ ECFCs were pre-treated with dimethyl sulfoxide or Compound $C(10 \mu M)$ for 30 min, then further incubated with or without VEGF $(25 \mathrm{ng} / \mathrm{mL})$ for $24 \mathrm{~h}$. Q-PCR analysis of mRNA levels of KLF2, eNOS, ICAM-1, CD31, CD34, and c-kit (C). Western blot analysis of protein levels of KLF2, eNOS, and phosphorylated eNOS (D). $\beta$-actin was an internal control. Data are mean \pm SD from three independent experiments, each performed in triplicate $(* P<0.05 ; * * P<0.01)$.

the down-stream regulator of the VEGF-AMPK signalling pathway governing ECFC function, similar to what has been reported in ECs. ${ }^{11}$

\subsection{KLF2 was required for ECFC differentiation}

To determine whether KLF2 contributes to ECFC differentiation, we used siRNA to silence KLF2 in ECFCs. The efficiency of siRNA in reducing KLF2 expression in ECFCs was confirmed by Q-PCR and western blot (Figure $3 A$ and $B$ ). The expression of TM and eNOS, two known KLF2 target genes, ${ }^{12,13}$ was also reduced, suggesting an effective inhibition of the function of KLF2 (Figure $3 A$ and $B$ ). Silencing of KLF2 decreased the expression of ICAM-1 but increased that of progenitor markers, which also suggests that KLF2 plays an important role in the process of ECFC differentiation (Figure 3B). The expression of ECFC surface markers was analysed by flow cytometry, which provided evidence that KLF2 was of importance in ECFC differentiation (Figure 3C). We further investigated the parameters of ECFC differentiation by analysing the role of KLF2 in ECFC tube-forming capacity. The in vitro tube-forming ability of ECFCs induced by VEGF was significantly inhibited with KLF2 knockdown (Figure 3D). Consistently, KLF2 knockdown impaired VEGF-induced migration of ECFCs, demonstrated by scratch-wound and trans-well assays (Figure $3 E$ and $F$ ).

\subsection{Overexpression of KLF2 enhanced ECFC differentiation}

To further explore the role of KLF2 in ECFC differentiation using a gain-of-function approach, we overexpressed KLF2 in ECFCs. ECFCs were infected with a FLAG-tagged adenovirus encoding full-length KLF2, and overexpression was confirmed by Q-PCR and western analysis (Figure 4A and B). Overexpression of KLF2 was accompanied by increased mRNA expression of the EC markers eNOS, CD31, and ICAM-1 and decreased expression of the stem/progenitor marker c-kit (Figure 4A). Interestingly, the mRNA level of CD34, another ECFC marker, was elevated (Figure 4A). Results were similar on flow cytometry of ECFC surface markers after KLF2 overexpression (Figure 4C). However, the tube-forming capacity was enhanced in ECFC overexpressing KLF2, when compared with cells infected with control adenovirus (Figure 4D). Thus, overexpression of KLF2 can mimic the effect of VEGF treatment and AMPK activation to promote ECFC differentiation. 

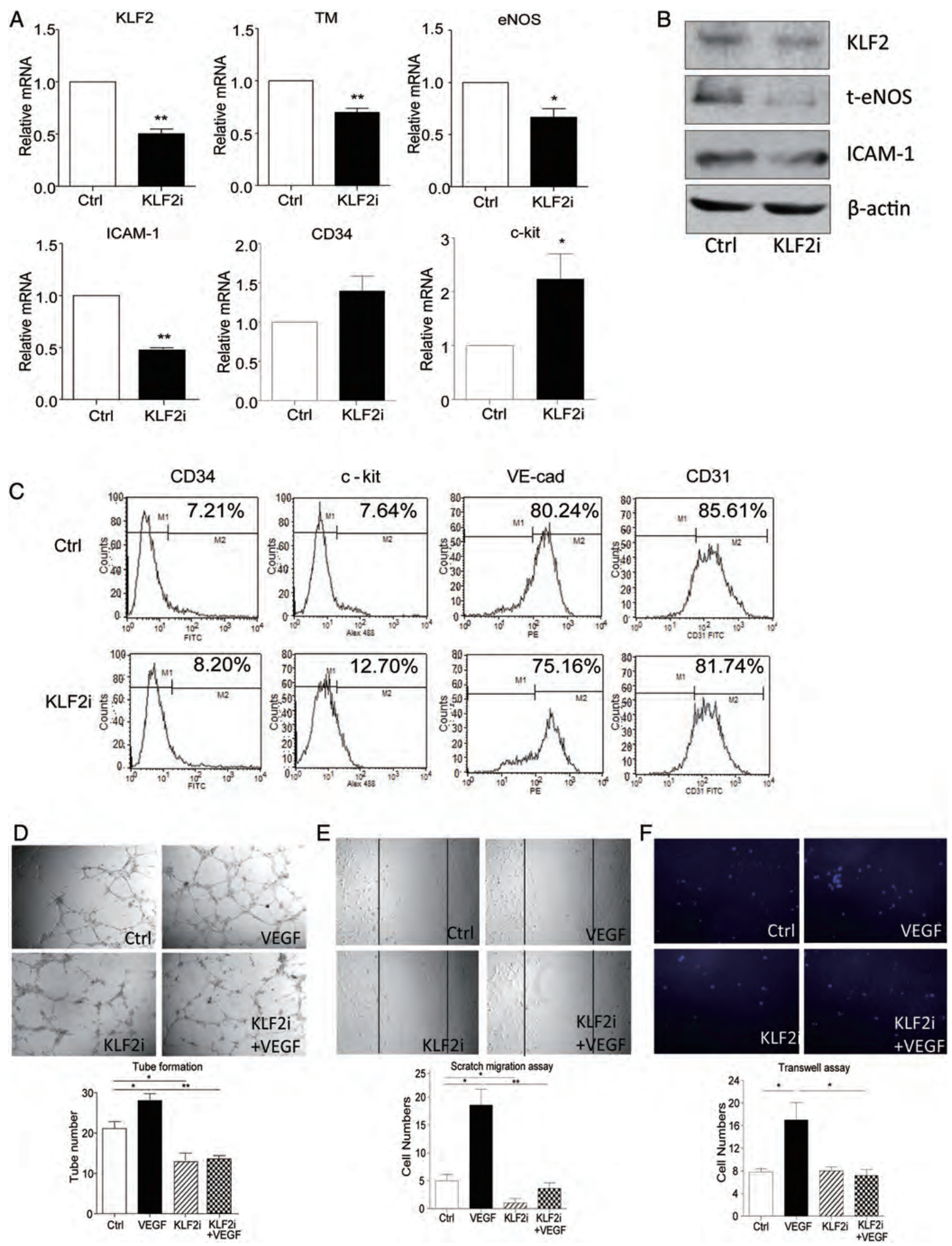

Figure 3 KLF2 knockdown impaired ECFC tube formation and migration. siRNA knockdown of KLF2 in ECFCs. (A and B) Q-PCR and western blot analysis of mRNA and protein levels of KLF2 and ECFC differentiation marker TM, eNOS, ICAM-1 CD34, and c-kit. $\beta$-actin was an internal control. (C) FACS analysis of ECFCs stained with antibodies against FITC-conjugated anti-human c-kit, FITC-conjugated anti-human CD34, FITC-conjugated antihuman CD31, PE-conjugated anti-human VE-cadherin. Data are representative of three separate experiments. (D-F) Control and knocked down cells were then cultured with or without VEGF $(25 \mathrm{ng} / \mathrm{mL})$ for $24 \mathrm{~h}$. Tube-formation $(D)$ and cell migration $(E$ and $F)$ assays were performed as described in Figure 1. Data are mean \pm SD from three independent experiments, each performed in triplicate $(* P<0.05 ; * * P<0.01)$. 

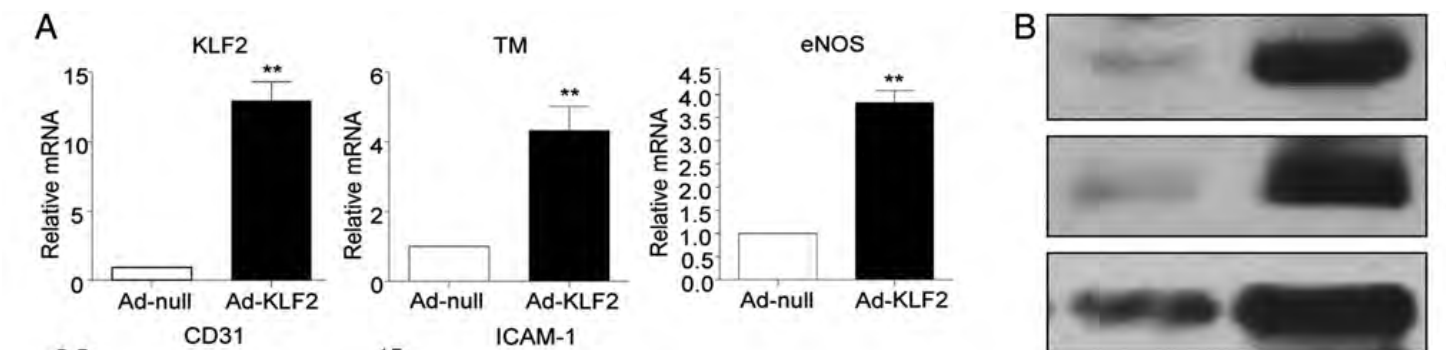

KLF2

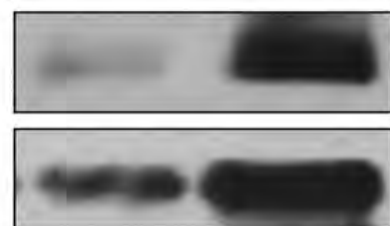

t-eNOS
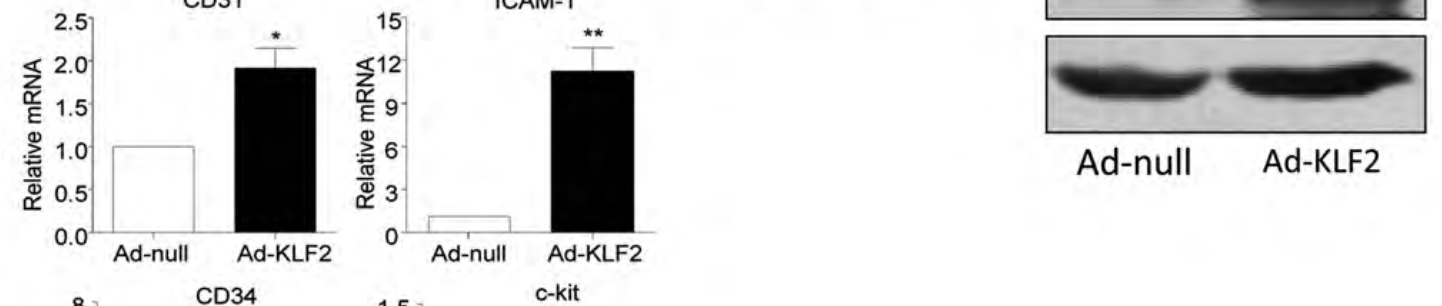

ICAM-1

Ad-null Ad-KLF2

\section{$\beta$-actin}
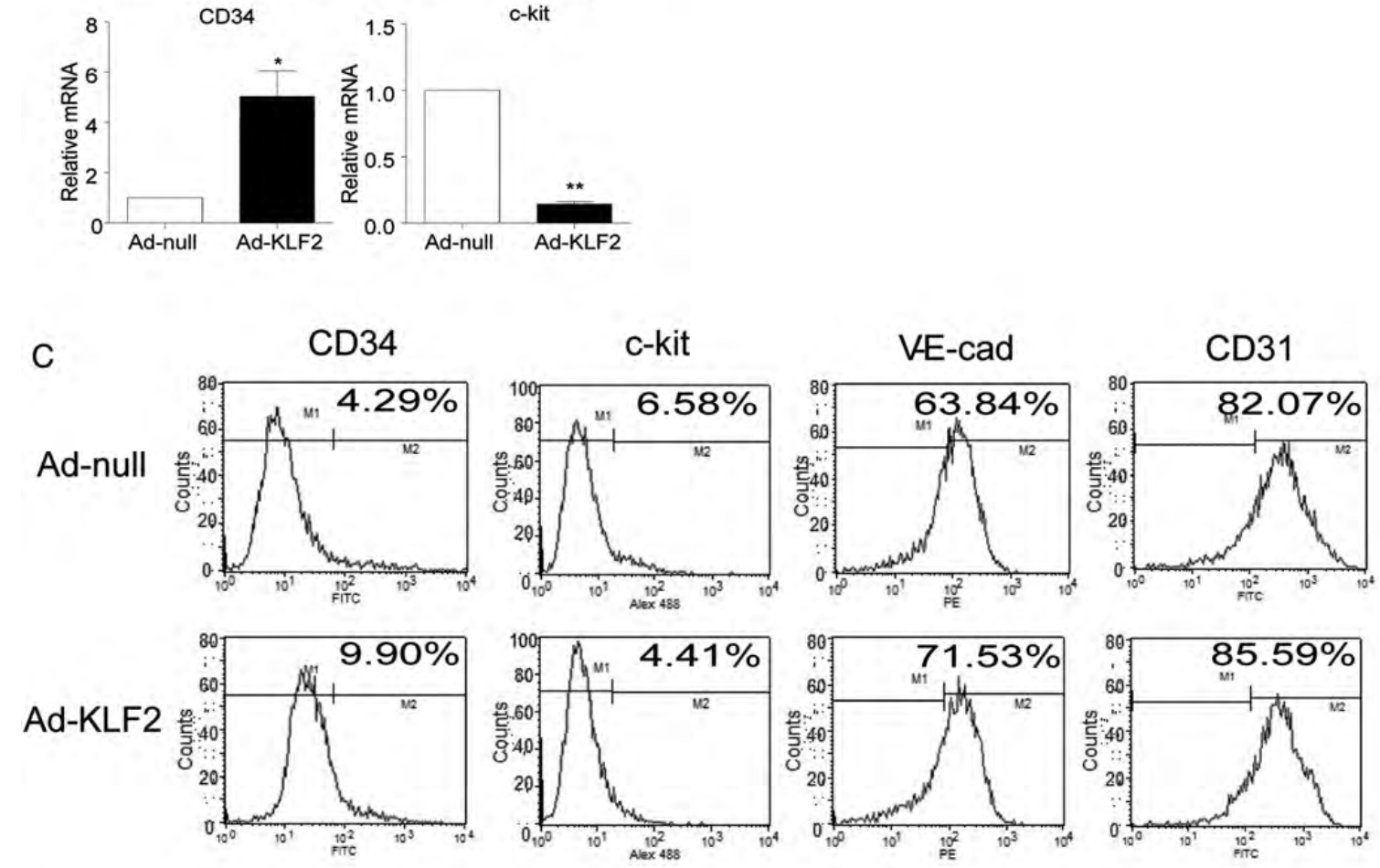

D
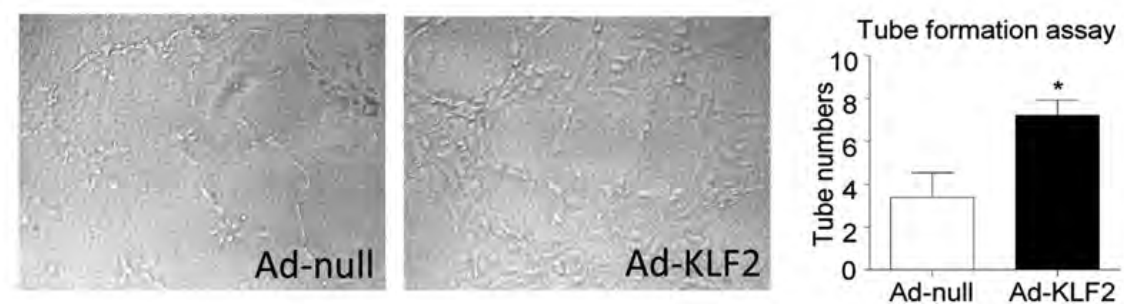

Figure 4 Overexpression of KLF2 drove ECFCs towards differentiation into ECs. ECFCs were infected with adenovirus overexpressing KLF2 (Ad-KLF2) or control vector (Ad-null) for 24 h. (A) Q-PCR analysis of mRNA levels of KLF2 and EC markers. (B) Western blot analysis of protein levels of KLF2, eNOS, and ICAM-1. (C) FACS analysis of ECFCs stained with antibodies against FITC-conjugated anti-human c-kit, FITC-conjugated anti-human CD34, FITCconjugated anti-human CD31, PE-conjugated anti-human VE-cadherin. Data are representative of three separate experiments. (D) Tube-formation assay was performed. Data are mean $\pm \mathrm{SD}$ from three independent experiments, each performed in triplicate $(* P<0.05 ; * * P<0.01)$. 

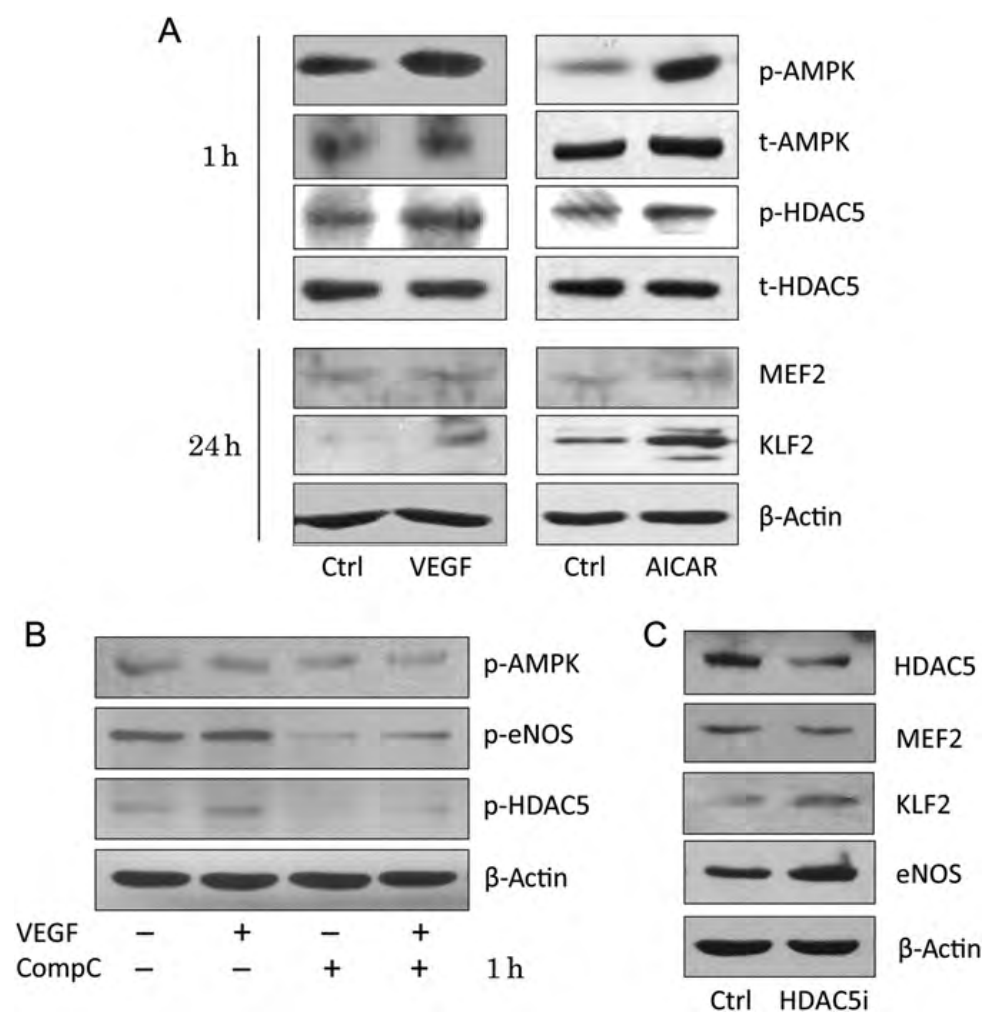

Figure 5 HDAC5 was involved in the regulation of ECFCs by the AMPK-KLF2 pathway. (A) ECFCs were incubated in the presence or absence of VEGF $(25 \mathrm{ng} / \mathrm{mL})$ and AICAR $(1 \mathrm{mM})$ for 1 or $24 \mathrm{~h}$, as indicated. Western blot analysis of levels of phosphorylated and unphosphorylated AMPK and HDAC5, MEF2, and KLF2. $\beta$-actin was an internal control. (B) ECFCs were pre-treated with dimethyl sulfoxide or Compound C (10 $\mu M$ ) for 30 min and further incubated with or without VEGF $(25 \mathrm{ng} / \mathrm{mL})$ for $1 \mathrm{~h}$. Western blot analysis of the levels of phosphorylated AMPK, eNOS, and HDAC5. $\beta$-actin was an internal control. (C) siRNA knockdown of HDAC5 in ECFCs. Western blot analysis of protein levels of eNOS, KLF2 MEF2, and HDAC5. $\beta$-actin was an internal control. Data are mean \pm SD from three independent experiments, each performed in triplicate $(* P<0.05 ; * * P<0.01)$.

\subsection{HDAC5 participated in the up-regulation of KLF2 by AMPK in ECFCs}

As a protein kinase, how does AMPK regulate KLF2, a transcription factor? MEF2 is a key transcription factor driving KLF2 expression, ${ }^{18}$ and HDAC5, a class Ila histone deacetylase, is a negative regulator of MEF2 transcriptional activity in cardiomyocytes and skeletal muscle cells, regulating muscle differentiation and cardiac growth. ${ }^{19,20}$ Moreover, HDAC5 can bind MEF2 and regulate its transcriptional activity but not its expression. In ECs, fluid shear stress stimulates the phosphorylation of HDAC5 and relieves its repression of MEF2, in turn permitting MEF2 activation and eNOS expression. ${ }^{21}$ We next investigated whether AMPK could stimulate HDAC5 phosphorylation and up-regulate KLF2 transcription in ECFCs. Both VEGF and AICAR induced HDAC5 phosphorylation but had no effect on MEF2 expression (Figure 5A). Compound $C$ abolished this effect (Figure 5B). In order to identify the role of HDAC5 in regulating KLF2, we further knocked down HDAC5 with HDAC5-specific siRNA. Loss of HDAC5 up-regulated both KLF2 and eNOS expression, with no change in MEF2 protein level (Figure 5C), which suggests that the AMPK-HDAC5-MEF2 pathway may mediate the up-regulation of KLF2 in ECFCs.

\subsection{KLF2 improved vasculogenesis by BMPC in vivo}

To further investigate the role of KLF2 in ECFC differentiation in vivo, we performed an in vivo angiogenesis assay with Matrigel plugs subcutaneously implanted in mice. BMPC from C57BL/6 mice were pretreated with VEGF, KLF2 adenovirus, or KLF2 siRNA suspended in Matrigel, and injected subcutaneously into C57BL/6 mice. After 7 days, Matrigel implants were removed, and haematoxylin and eosin staining revealed a greater number of luminal structures in implants containing KLF2-overexpressing cells compared with cells infected with control adenovirus. In contrast, cells treated with KLF2 siRNA exhibited a poor tube formation capacity (Figure 6A). To further characterize the differentiation of these BMPCs, immunohistochemical staining of implants showed cells in luminal structures positive for VE-cadherin, a marker of mature ECs (Figure 6B). The number of VE-cadherin positive structures was significantly higher with KLF2 overexpression than control treatment. In contrast, KLF2-siliencing reduced the number of VE-cadherin-positive structures. VEGF-treatment was used as positive control (Figure 6A and B). Analysis of the FLAG-tagged KLF2 adenovirus identified tube structures in cells from the donor mice (Figure 6C). Overexpression of KLF2 after 7 days still persisted in Ad-KLF2 treated BMPCs but was not detectable in control cells (Figure 6C, see Supplementary material online, Figure S2).

\section{Discussion}

The KLF proteins are a family of zinc finger-containing transcription factors that regulate proliferation, differentiation, development, and programmed cell death during vascular gene expression. KLF2 is an 

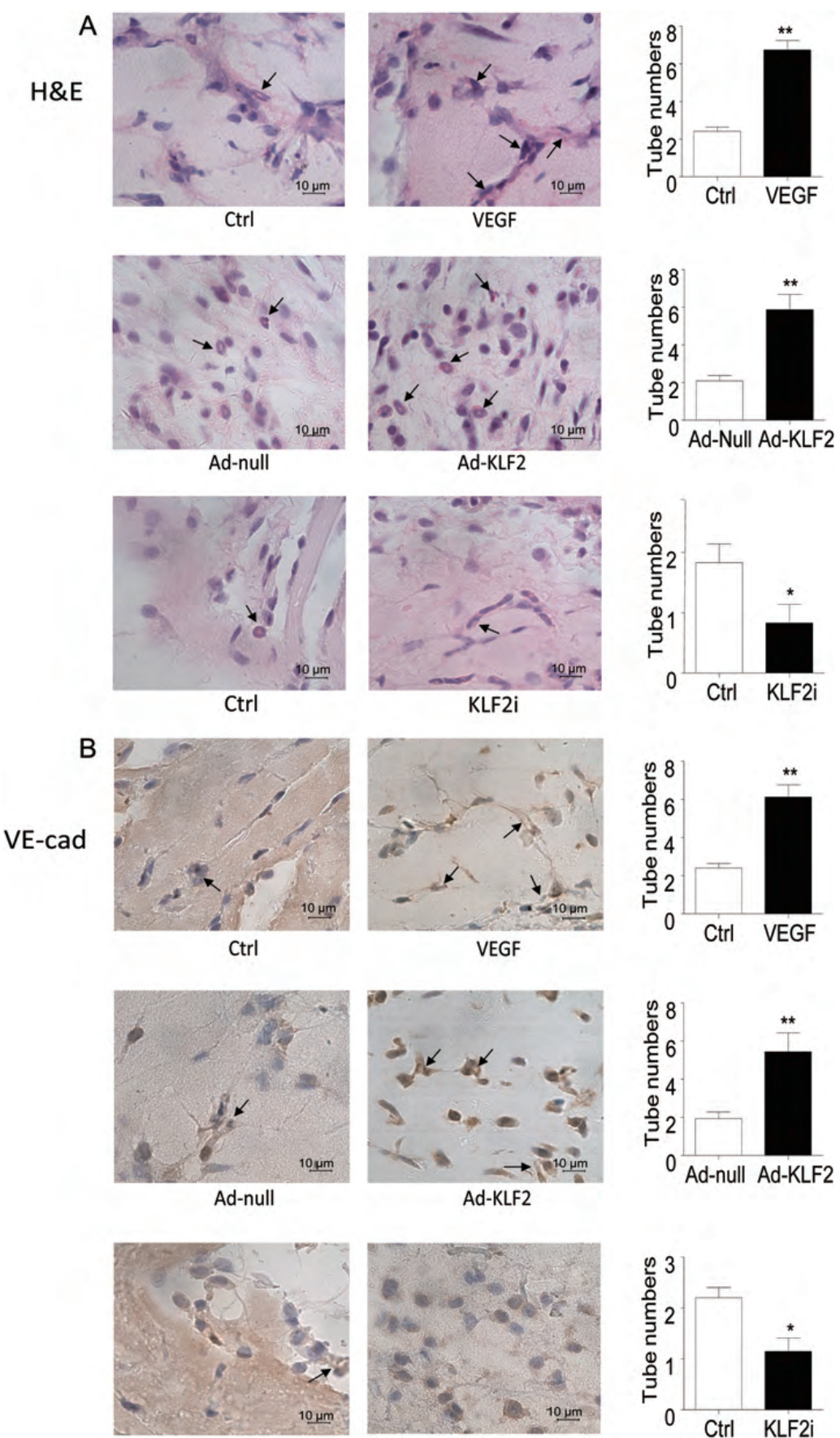

Ctrl
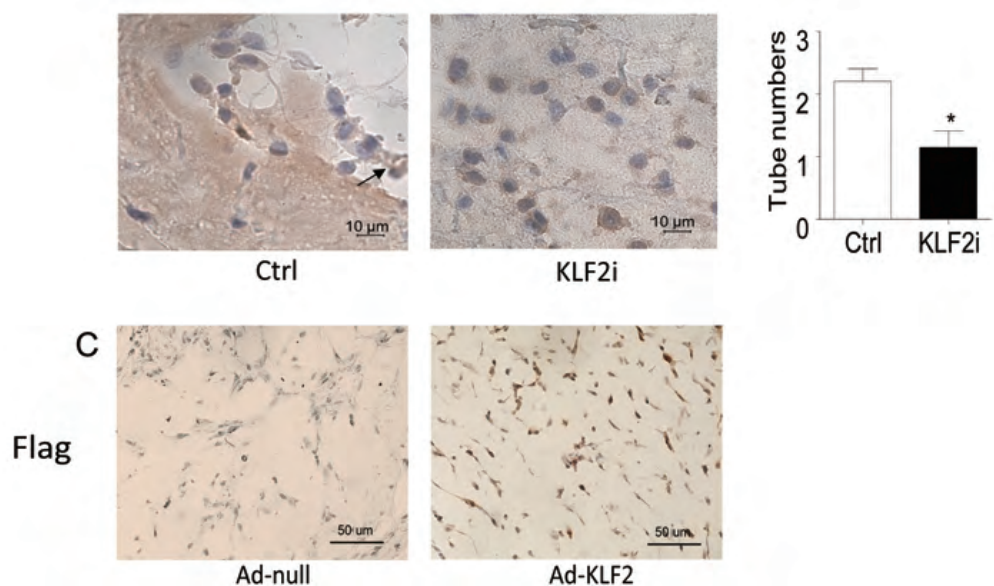

Figure 6 Neovascularization by BMPC in mice was enhanced by KLF2 overexpression. BMPC from C57BL/6 mice were infected with adenovirus encoding KLF2 (Ad-KLF2, 50 multiplicity of infection) or with siRNA knockdown of KLF2 and mixed with Matrigel. Cells were subcutaneously injected into C57BL/6 mice, which were maintained for 7 days. Implants were sectioned and stained with haematoxylin and eosin (H\&E) (A), anti-VE-cadherin (VE-Cad) (B), and anti-FLAG (Flag) (C). Arrows indicate vessel-like structures. Images are representative of implants from six different animals. Microvessel density in Matrigel implants was quantified by counting luminal structures containing erythrocytes. Data are mean \pm SD from three independent experiments, each performed in triplicate $(* P<0.05 ; * * P<0.01)$. Magnification, $\times 630(A$ and $B), \times 400(C)$. 
important factor in embryonic development, particularly cardiovascular development, because loss of KLF2 causes lethal cardiac failure at E12.5-E14.5. ${ }^{22,23}$ KLF2 was also reported to modulate angiogenesis, the process of new blood vessel formation; ${ }^{16,24}$ however, its role in angiogenesis is controversial. ${ }^{25}$ Here, we investigated the effect of KLF2 on EPC differentiation and function, and the underlying mechanism. We found (i) KLF2 induced the differentiation and migration of EPCs, which was followed by up-regulation of the mature EC markers; (ii) KLF2 promoted neovascularization in vitro and in vivo; and (iii) differentiation of EPCs was mediated by the AMPK-HDAC5-KLF2-eNOS signalling pathway. The summary scheme of our findings combined with our previous reports showed in Supplementary material online, Figure S3.

EPC, referring to cells that display cell surface antigens similar to ECs in vivo, logged in areas of vascular injury and repaired damaged blood vessels. Many sources of EPC have been reported. EPCs derived from bone marrow, peripheral blood, or resident tissues ${ }^{26}$ play key roles in the treatment of cardiovascular ischaemic diseases. Most subpopulations of EPCs reported are now known to be members of haemopoietic lineage. Richardson and Yoder $^{27}$ reviewed ECFC functioned as precursor cells which displayed the most features of a human postnatal vasculogenic cells was other subpopulation of pro-angiogenic progenitor cells. In this study, we used umbilical blood-derived cobblestone-like cells which were found to match the definition of ECFC and BMPCs for studying the role of KLF2 in EPCs.

KLF2 plays an important role in T-cell differentiation ${ }^{28}$ and vascular development. ${ }^{16}$ Increasing evidence suggests that KLF2 plays a critical role in vascular biology, $8,15,29$ because KLF2 has recently been identified as a flow-responsive gene. ${ }^{15,29}$ Moreover, there have also been reports that KLF2 is an anti-atherosclerotic and anti-inflammatory regulator via KLF2-induced increases in eNOS expression and activity in response to various pro-inflammatory cytokines, ${ }^{30,31}$ further suggesting that KLF2 participates in the regulation of vascular biology and endothelial function. It was reported that KLF2 inhibited VEGF-A-mediated angiogenesis in ECs, indicating KLF2 was anti-angiogenic. ${ }^{25}$ However, overexpression of KLF2 increased eNOS expression in circulating pro-angiogenic cells in vitro and augments neovascularization in bone-marrow-derived mononuclear cells in a murine hind-limb ischaemia model. ${ }^{24}$ In consistent with this report, we found that KLF2 promoted the differentiation of EPCs into mature ECs, the cells possess the postnatal vasculogenic ability. The discrepancy between the studies indicated that KLF2 played different roles in different type of cells and development stage.

In the current study, we have evaluated the role of KLF2 in EPC biology using both loss- and gain-of-function approaches (Figures 3 and 4). We have demonstrated EPCs from either human cord blood or mouse bone marrow had neovascularization capacity regulated by KLF2 in vitro and in vivo (Figures 4 and 6 ). The up-regulation of mature endothelial markers, including eNOS, induced by overexpression of KLF2, as well as the increased capacity for tube formation, strongly suggest that KLF2 induces ECFC differentiation towards the endothelial lineage (Figures 3 and 4). The stem/progenitor marker c-kit was also down-regulated after KLF2 overexpression and up-regulated with KLF2 knockdown, indicating that KLF2 was an inducer of endothelial differentiation (Figures 3 and 4). Interestingly, CD34, another known progenitor marker, showed an opposite expression panel to that of c-kit, in response to KLF2. This result suggested that CD34 may not be used as a sole progenitor marker. The possible explanation is that human CD34 might be a target gene of KLF2 since MatInspector promotor analysis software (Online software: http://www.genomatix.de/online_help/help_mat inspector/matinspector_help.html) gave a hint that there was a binding site of KLFs in the promoter region of CD34. Papers have reported that members of the KLF family had similar bio-functions. ${ }^{29,32}$ In light of the cross-talk of the KLFs family, combined with the results of overexpression experiments, KLF2 might be a transcriptional regulator of CD34.

We previously reported that the activation of AMPK by VEGF and statins promoted the differentiation of human cord blood-derived EPCs into mature ECs via a nitric oxide-dependent mechanism. ${ }^{5}$ Flow-induced AMPK activation was reported to up-regulate the expression of KLF2 in ECs. ${ }^{11}$ However, our use of LIF to maintain the undifferentiated status of progenitor cells activated Akt and up-regulation of KLF4. The effect of LIF on KLF4 could be blocked by overexpression of dominant-negative Akt adenovirus. ${ }^{33}$ In this study, treating ECFCs with VEGF to promote ECFC differentiation to mature ECs upregulated the expression of KLF2, accompanied by up-regulation of its target genes, including eNOS. AMPK activation apparently plays an important role in this process because AMPK agonist AICAR mimicked, and antagonist Compound $C$ attenuated the effect of VEGF. Thus, it appears that AMPK activation mediates up-regulation of KLF2 expression and promotion of cell differentiation to mature ECs. In contrast, Akt activation drove EPC proliferation and maintained EPC stemness. The balance of activation of AMPK and Akt may provide new insights into the regulation of the properties of EPCs.

To further explore the mechanism underlying AMPK regulation of KLF2, we investigated the pathway described in ECs, whereby AMPK regulates KLF2 via HDAC5 phosphorylation and MEF2 activation. ${ }^{11,21}$ We found AMPK activation induced HDAC5 phosphorylation, which indirectly up-regulated KLF2 expression in ECFCs (Figure 5). However, other mechanisms involved in the regulation of HDAC or posttranscriptional regulation cannot be excluded by the current study. KLF2 reinforced the process of angiogenesis by promoting the differentiation of EPCs to mature ECs and, thus constitutes a potential novel target for clinical therapy in ischaemic heart diseases.

\section{Supplementary material}

Supplementary material is available at Cardiovascular Research online.

\section{Conflict of interest: none declared.}

\section{Funding}

This work was supported in part by grants from the National Natural Science Foundation of China (nos 81070113, 30600209, 30630032, 30821001) and the Major National Basic Research Grant of China (no. 2010CB912504).

\section{References}

1. Gomez-Cerezo JF, Pagan-Munoz B, Lopez-Rodriguez M, Estebanez-Munoz M, Barbado-Hernandez FJ. The role of endothelial progenitor cells and statins in endothelial function: a review. Cardiovasc Hematol Agents Med Chem 2007;5:265-272.

2. Hirschi KK, Ingram DA, Yoder MC. Assessing identity, phenotype, and fate of endothelial progenitor cells. Arteriosclerosis, thrombosis, and vascular biology 2008;28:1584-1595.

3. Takahashi T, Kalka C, Masuda H, Chen D, Silver M, Kearney M et al. Ischemia- and cytokine-induced mobilization of bone marrow-derived endothelial progenitor cells for neovascularization. Nat Med 1999;5:434-438.

4. Xiao-Yun X, Zhao-Hui M, Ke C, Hong-Hui H, Yan-Hong X. Glucagon-like peptide-1 improves proliferation and differentiation of endothelial progenitor cells via upregulating VEGF generation. Med Sci Monit 2011;17:BR35-BR41.

5. LiX, Han Y, Pang W, Li C, Xie X, Shyy JY et al. AMP-activated protein kinase promotes the differentiation of endothelial progenitor cells. Arterioscler Thromb Vasc Biol 2008;28: 1789-1795.

6. Zhu Z, Fu C, Li X, Song Y, Li C, Zou M et al. Prostaglandin E2 promotes endothelial differentiation from bone marrow-derived cells through AMPK activation. PLoS One 2011;6: e23554. 
7. McConnell BB, Yang VW. Mammalian Kruppel-like factors in health and diseases. Physiol Rev 2010;90:1337-1381.

8. Gracia-Sancho J, Villarreal G Jr, Zhang Y, Garcia-Cardena G. Activation of SIRT1 by resveratrol induces KLF2 expression conferring an endothelial vasoprotective phenotype. Cardiovasc Res 2010;85:514-519.

9. Egorova AD, Van der Heiden K, Van de Pas S, Vennemann P, Poelma C, DeRuiter MC et al. Tgfbeta/Alk5 signaling is required for shear stress induced klf2 expression in embryonic endothelial cells. Dev Dyn 2011;240:1670-1680.

10. Sako K, Fukuhara S, Minami T, Hamakubo T, Song H, Kodama T et al. Angiopoietin-1 induces Kruppel-like factor 2 expression through a phosphoinositide 3-kinase/AKTdependent activation of myocyte enhancer factor 2.J Biol Chem 2009;284:5592-5601.

11. Young A, Wu W, Sun W, Benjamin Larman H, Wang N, Li YS et al. Flow activation of AMP-activated protein kinase in vascular endothelium leads to Kruppel-like factor 2 expression. Arterioscler Thromb Vasc Biol 2009;29:1902-1908.

12. Lin Z, Kumar A, SenBanerjee S, Staniszewski K, Parmar K, Vaughan DE et al. Kruppellike factor 2 (KLF2) regulates endothelial thrombotic function. Circ Res 2005;96: e48-e57.

13. Parmar KM, Larman HB, Dai G, Zhang Y, WangET, Moorthy SN et al. Integration of flowdependent endothelial phenotypes by Kruppel-like factor 2. J Clin Invest 2006;116: 49-58.

14. Atkins GB, Wang Y, Mahabeleshwar GH, Shi H, Gao H, Kawanami D et al. Hemizygous deficiency of Kruppel-like factor 2 augments experimental atherosclerosis. Circ Res 2008; 103:690-693.

15. Boon RA, Horrevoets AJ. Key transcriptional regulators of the vasoprotective effects of shear stress. Hamostaseologie 2009;29:39-40. 41-33.

16. Meadows SM, Salanga MC, Krieg PA. Kruppel-like factor 2 cooperates with the ETS family protein ERG to activate Flk1 expression during vascular development. Development 2009;136:1115-1125.

17. Wang L, Zheng J, Bai X, Liu B, Liu CJ, Xu Q et al. ADAMTS-7 mediates vascular smooth muscle cell migration and neointima formation in balloon-injured rat arteries. Circ Res 2009;104:688-698.

18. Kato Y, Kravchenko VV, Tapping RI, Han J, Ulevitch RJ, Lee JD. BMK1/ERK5 regulates serum-induced early gene expression through transcription factor MEF2C. EMBO J 1997; 16:7054-7066.

19. McKinsey TA, Zhang CL, Lu J, Olson EN. Signal-dependent nuclear export of a histone deacetylase regulates muscle differentiation. Nature 2000;408:106-111.
20. McKinsey TA, Zhang CL, Olson EN. Activation of the myocyte enhancer factor-2 transcription factor by calcium/calmodulin-dependent protein kinase-stimulated binding of 14-3-3 to histone deacetylase 5. Proc Natl Acad Sci USA 2000;97:14400-14405.

21. Wang $\mathrm{W}, \mathrm{Ha} \mathrm{CH}$, Jhun $\mathrm{BS}$, Wong $\mathrm{C}$, Jain MK, Jin ZG. Fluid shear stress stimulates phosphorylation-dependent nuclear export of HDAC5 and mediates expression of KLF2 and eNOS. Blood 2010;115:2971-2979.

22. Kuo CT, Veselits ML, Barton KP, Lu MM, Clendenin C, Leiden JM. The LKLF transcription factor is required for normal tunica media formation and blood vessel stabilization during murine embryogenesis. Gene Dev 1997;11:2996-3006.

23. Wani MA, Means RT Jr, Lingrel JB. Loss of LKLF function results in embryonic lethality in mice. Transgenic Res 1998;7:229-238.

24. Boon RA, Urbich C, Fischer A, Fontijn RD, Seeger FH, Koyanagi M et al. Kruppel-like factor 2 improves neovascularization capacity of aged proangiogenic cells. Eur Heart J 2011;32:371-377.

25. Bhattacharya R, Senbanerjee S, Lin Z, Mir S, Hamik A, Wang P et al. Inhibition of vascular permeability factor/vascular endothelial growth factor-mediated angiogenesis by the Kruppel-like factor KLF2. J Biol Chem 2005;280:28848-28851.

26. Kirton JP, Xu Q. Endothelial precursors in vascular repair. Microvasc Res 2010;79: 193-199.

27. Richardson MR, Yoder MC. Endothelial progenitor cells: quo vadis? J Mol Cell Cardiol 2011;50:266-272.

28. Carlson CM, Endrizzi BT, Wu J, Ding X, Weinreich MA, Walsh ER et al. Kruppel-like factor 2 regulates thymocyte and T-cell migration. Nature 2006;442:299-302.

29. Atkins GB, Jain MK. Role of Kruppel-like transcription factors in endothelial biology. Circ Res 2007;100:1686-1695.

30. Tuomisto TT, Lumivuori H, Kansanen E, Hakkinen SK, Turunen MP, van Thienen JV et al. Simvastatin has an anti-inflammatory effect on macrophages via upregulation of an atheroprotective transcription factor, Kruppel-like factor 2. Cardiovasc Res 2008;78: $175-184$.

31. Arkenbout EK, Dekker RJ, de Vries CJ, Horrevoets AJ, Pannekoek H. Focusing on transcription factor families in atherogenesis: the function of LKLF and TR3. Thromb Haemost 2003;89:522-529.

32. Hall J, Guo G, Wray J, Eyres I, Nichols J, Grotewold L et al. Oct4 and LIF/Stat3 additively induce Kruppel factors to sustain embryonic stem cell self-renewal. Cell Stem Cell 2009;5: 597-609.

33. Li X, Song Y, Wang D, Fu C, Zhu Z, Han Y et al. LIF maintains progenitor phenotype of endothelial progenitor cells via Kruppel-like factor 4. Microvasc Res 2012;84:270-277. 